\title{
Mapping the Role of Digital Health Technologies in Prevention and Control of COVID-19 Pandemic: Review of the Literature
}

\author{
Binyam Tilahun', Kassahun Dessie Gashu', Zeleke Abebaw Mekonnen"1, ${ }^{1}$, Berhanu Fikadie \\ Endehabtu', Dessie Abebaw Angaw ${ }^{3}$ \\ 1 Department of Health Informatics, Institute of Public Health, College of Medicine and Health \\ Sciences, University of Gondar, Gondar, Ethiopia \\ 2 Health System Directorate, Ministry of Health, Ethiopia \\ 3 Department of Epidemiology and Biostatistics, Institute of Public Health, College of Medicine and \\ Health Sciences, University of Gondar, Gondar, Ethiopia
}

\begin{abstract}
Summary
Background: Coronavirus Disease (COVID-19) is currently spreading exponentially around the globe. Various digital health technologies are currently being used as weapons in the fight against the pandemic in different ways by countries. The main objective of this review is to explore the role of digital health technologies in the fight against the COVID-19 pandemic and address the gaps in the use of these technologies for tackling the pandemic.

Methods: We conducted a scoping review guided by the Joanna Briggs Institute guidelines. The articles were searched using electronic databases including MEDLINE (PubMed), Cochrane Library, and Hinari. In addition, Google and Google scholar were searched. Studies that focused on the application of digital health technologies on COVID-19 prevention and control were included
\end{abstract}

in the review. We characterized the distribution of technological applications based on geographical locations, approaches to apply digital health technologies and main findings. The study findings from the existing literature were presented using thematic content analysis.

Results: A total of 2,601 potentially relevant studies were generated from the initial search and 22 studies were included in the final review. The review found that telemedicine was used most frequently, followed by electronic health records and other digital technologies such as artificial intelligence, big data, and the internet of things (loT). Digital health technologies were used in multiple ways in response to the COVID-19 pandemic, including screening and management of patients, methods to minimize exposure, modelling of disease spread, and supporting overworked providers.
Conclusion: Digital health technologies like telehealth, mHealth, electronic medical records, artificial intelligence, the internet of things, and big data/internet were used in different ways for the prevention and control of the COVID-19 pandemic in different settings using multiple approaches. For more effective deployment of digital health tools in times of pandemics, development of a guiding policy and standard on the development, deployment, and use of digital health tools in response to a pandemic is recommended.

\section{Keywords}

COVID-19, digital health, pandemics, technology, review

Yearb Med Inform 2021:26-37

http://dx.doi.org/10.1055/s-0041-1726505

\section{Background}

In recent decades, the world has seen a dramatic rise in global pandemics, from the Severe Acute Respiratory Syndrome (SARS) pandemic in 2003 to Avian Influenza in 2006, H1N1 influenza in 2009, Middle East Respiratory Syndrome (MERS) in 2012, Ebola in 2014, the appearance of the Zika virus in Latin America in 2015. These pandemics are inextricably bound up in modern socio-technical developments and processes of globalization [1-3]. In early 2020, the World Health Organization declared a new pandemic, caused by Severe Acute Respiratory Syndrome Coronavirus-2 (SARS-CoV-2), a previously unknown SARS virus variant $[1,4]$. SARS-CoV-2 causes the infectious disease known as COVID-19. Characteristic of a pandemic in general, COVID-19 is spreading around the globe exponentially, causing considerable disruption not only on the health system but also on the world economy. Compared with SARS and MERS, COVID-19 spreads more rapidly owing to increased globalization, a longer incubation period, and asymptomatic transmission.

The management and control of pandemics represent a unique challenge to health systems because novel infectious diseases can be transmitted rapidly to others and thus require a focus on early detection and treat- ment, surveillance, and outbreak control. Applications of digital health technologies like artificial intelligence, telehealth, mobile health, big data, $5 \mathrm{G}$, and the Internet of Things (IoT) may be useful to support the control of a pandemic $[5,6]$.

Digital health technologies (DHTs) have played an important role in pandemics, aiding understanding of the disease, improving diagnostic tools, and controlling the spread of the infection [4]. DHTs bring health services directly to people's homes and underserved communities that make health care more responsive and productive [6]. It has been reported that DHTs such as telehealth, when used for health surveys and data collec- 
tion, surveillance, health awareness-raising, and decision support systems from different sources can be used to prevent and control pandemics or outbreaks without disrupting the regular social order $[7,8]$. The use of digital tools such as contact tracing apps to monitor outbreaks and their extent, and online consultations to help keep health professionals and patients safe while providing continued care are some of the ways that the potential of digital health has been harnessed during the COVID-19 pandemic [9].

The COVID-19 pandemic has challenged health systems in many ways and it is critically important to fight COVID-19 effectively. In line with this, digital health solutions provide an opportunity to optimize time and human resources in the fight against the pandemic. These solutions facilitate communication between community members, hospitals, and health care providers [10]. Yet, there are few reviews for how DHTs contribute to pandemics, especially COVID-19. A rigorous scoping review that examines the role of DHT in preventing and controlling the COVID-19 pandemic is needed to examine how DHTs were rapidly deployed to support COVID-19 response.

We used a Population, Concept, and Context (PCC) framework developed by the Joanna Briggs Institute (JBI) to frame our primary research questions as follows:

1. What is the available evidence on the role of digital health technologies in the prevention and control of COVID-19?

2. What were the barriers that influence the practice of digital health technologies in the prevention and control of the COVID -19 pandemic?

To answer these questions, we aimed to map the evidence of digital health technologies on COVID-19 pandemic control and barriers during the application of these technologies.

\section{Methods}

Our scoping review aimed to map the available evidence on the role of DHTs, such as electronic health records, mHealth, telehealth, and artificial intelligence for preventing and controlling COVID-19.

Table 1 Lists of the inclusion and exclusion criteria of studies for the scoping review

\begin{tabular}{|l|l|}
\hline Inclusion criteria & Exclusion criteria \\
\hline - Both quantitative and qualitative studies & - Case reports \\
- Studies related to the role of digital health technologies & - Individual views or "perspective" pieces \\
to manage the COVID-19 pandemic & - Literature reviews \\
- Studies published in the English language & - Studies published in a language other than English \\
- Studies published in peer-reviewed journals & - Studies published in preprint literature \\
\hline
\end{tabular}

This review was conducted within the Reporting Items for Systematic Reviews and Meta-Analyses extension for Scoping Reviews (PRISMA-ScR) checklist and it was guided by the Joanna Briggs Institute scoping review guidance [11].

\subsection{Eligibility Criteria}

See Table 1.

\subsection{Data Sources and Search Strategy}

A comprehensive search was performed on the following databases: MEDLINE (PubMed), Cochrane library, Hinari ${ }^{1}$, Google, and Google Scholar advanced databases. Medical Subject Headings (MeSH) terms were used for searching and Boolean operators (AND, OR) were used to specify combinations of search terms. The following keywords and medical subject headings were used:

- For coronavirus: COVID-19 OR "coronavirus pandemic" OR pandemic OR "public health epidemics" OR "global pandemics" OR "disease outbreaks" OR coronavirus OR "coronavirus 2019" OR "novel coronavirus disease 2019" OR SARS-CoV-2

Hinari Programme set up by World Health Organization together with major publishers, enables low- and middle- income countries to gain access to one of the world's largest collections of biomedical and health literature. (https://www.who. int/hinari/en/)
AND

- For digital health technologies: "health informatics" OR "clinical informatics" OR "(computer science, medical)" OR "health information technology" OR informatics OR "medical computer science" OR "medical records systems" OR "electronic health records" OR "biomedical informatics community" OR telemedicine OR telemed OR "telehealth eHealth" OR mHealth OR "electronic health records" OR "electronic medical record" OR "patient-facing technology"

\section{AND}

\section{- Prevention OR control OR Management.}

The search was performed from October 09, 2020 to October 12,2020. Additional relevant articles were identified by searching the reference lists of full-text articles studies published up to the last searched date of October 12, 2020, were included (Supplement File 1).

Initial searches were performed by an author (DA), who has experience in systematic reviews. Screening of titles, abstracts, and full texts was conducted independently by two authors (DA and $\mathrm{KD})$. In this process, a second reviewer was blinded to the primary reviewer's decision for selecting the articles; this helped to mitigate the risk of bias during the assessment stages of the reviews. Any differences of opinion were discussed, and a consensus was reached; otherwise, a third reviewer (BT) was involved to arbitrate any issues that remained unresolved. 


\subsection{Data Extraction}

Two authors (DA and BF) extracted the data independently and compared the results. We extracted the following data from each study: author name, year of publication, country, types of digital health technology used, purpose(s) of applying digital health technology, service users, study design, approaches to applications of digital health technologies to address COVID-19, the study main findings, and barriers to implementation or adoption (Table 1).

\subsection{Analysis}

Based on the methodological framework for scoping reviews [12], we were able to present our narrative account of findings in two ways. First, attention was given to the basic numerical analysis, and the distribution of the studies included in the review. We determined the distribution of electronic technologies used in COVID-19 prevention and control by geographical location. Second, the study findings from the existing literature were evaluated using thematic content analysis. Our narrative was then structured around the categories derived from the included study results or outcomes. These categories were: 1) types of commonly used digital health technologies, approaches or methods of application of digital health technologies for pandemic prevention and control; 2) the main role of the digital health technologies; and 3) barriers for the proper use of digital health technologies to prevent and control the pandemic.

\section{Results}

The initial search generated 2,601 articles. After duplicates were excluded, 2,431 articles remained. Titles and abstracts of the remaining articles were screened for potential eligibility. We excluded most of these potentially relevant papers $(89.6 \%)$ based on a review of the title $(n=1,188)$ or abstract ( $n=991)$. As a result, a total of 252 articles were eligible for full-text screening. After screening, 230 articles were excluded due to: lack of relevant information $(n=74$,
$32.2 \%$ ), for example, no information about DHTs rather provide information only about COVID-19 and other infectious diseases; electronic technologies applied for other types of disease such as HIV/AIDS, tuberculosis, or diabetes mellitus ( $n=99,43 \%)$; focus only on health information technology in general, such as for communication and academic education $(n=36,15.7 \%)$. Another $21(9.1 \%)$ documents, representing perspective articles or literature reviews were excluded (Figure 1).

\subsection{Characteristics of Included Studies}

Of the 22 included studies 13 (59\%) of them were from the USA [6, 13-24], three were from China [25-27], two were from Iran [28, 29], Australia [16], Spain [30], Canada [31], and Taiwan [32] each contributed one (Table 2 ). The use of digital health technologies to combat COVID-19 was predominantly reported by North American studies (64\%), followed by Asia (27\%).

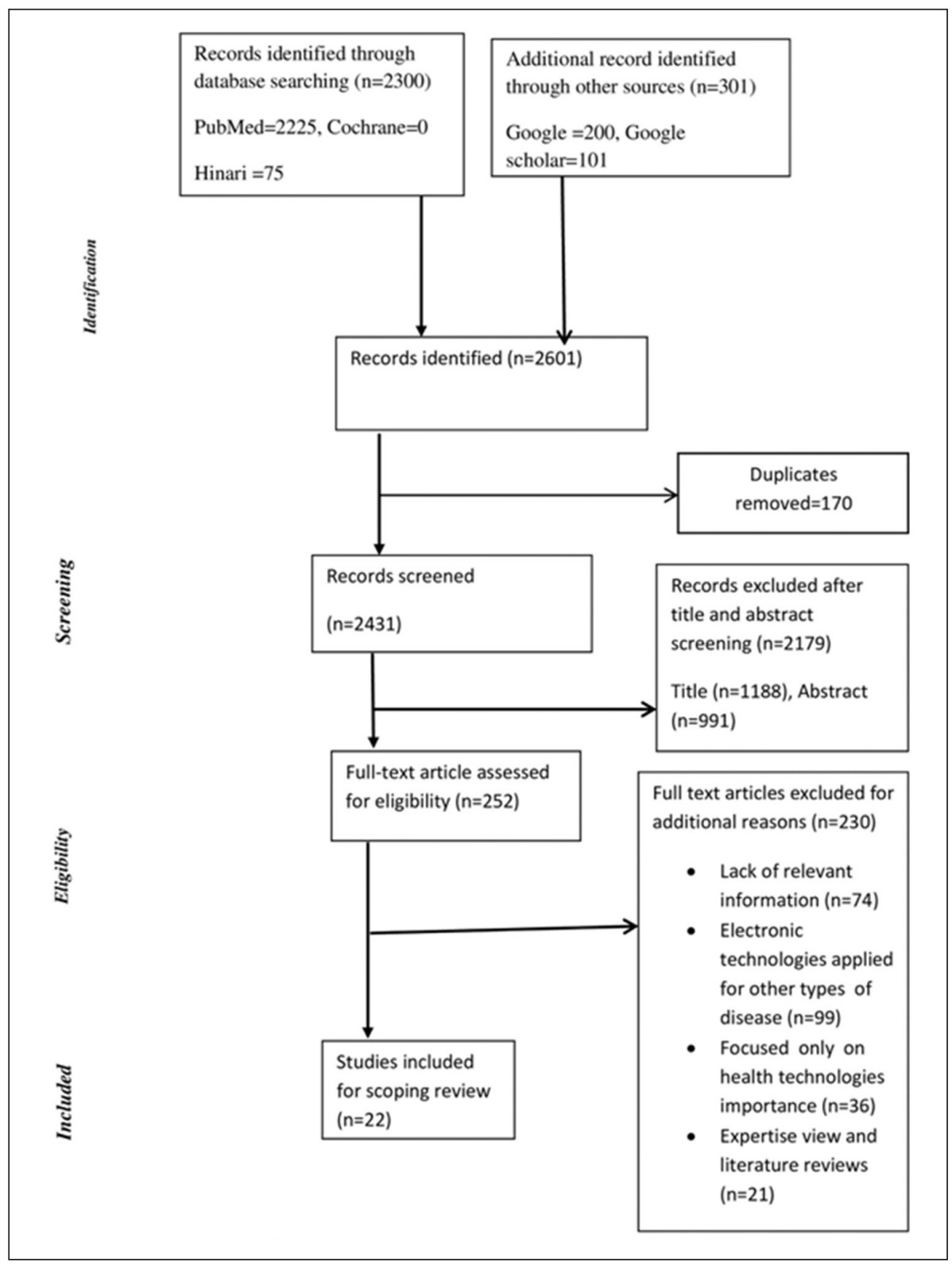

Fig. 1 Flow Diagram for the scoping review process adapted from the PRISMA statement by Moher and colleagues (2009) 


\begin{tabular}{|c|c|c|c|c|c|}
\hline 鵕 & 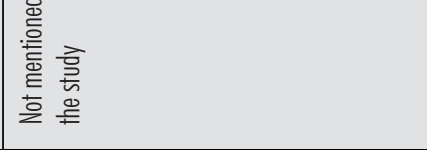 & 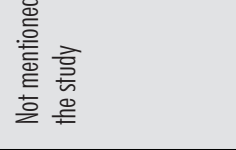 & 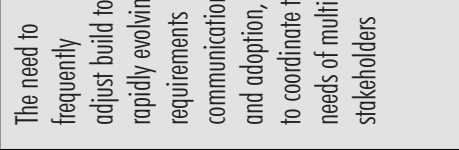 & 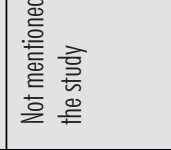 & 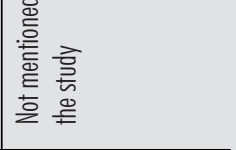 \\
\hline & 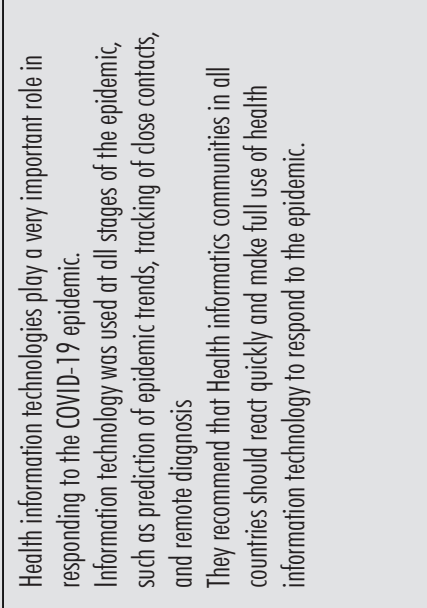 & 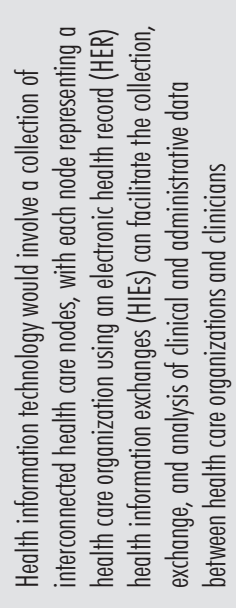 & 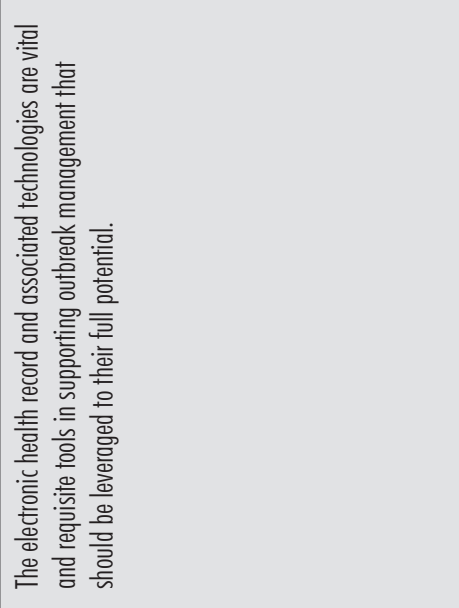 & 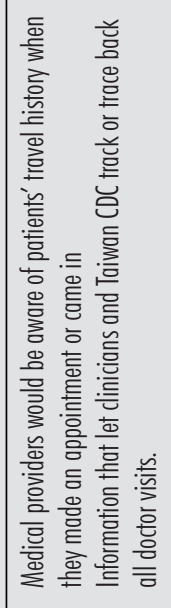 & 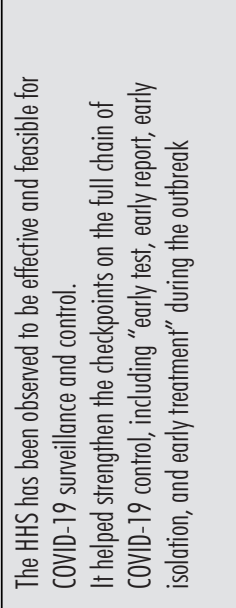 \\
\hline & 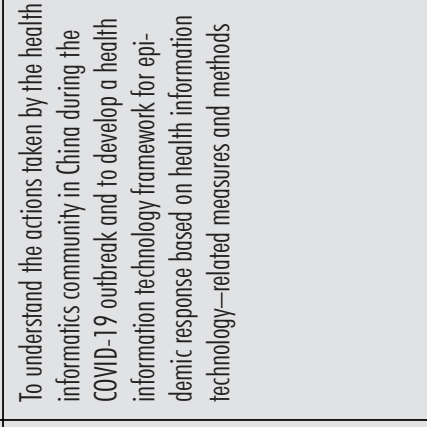 & 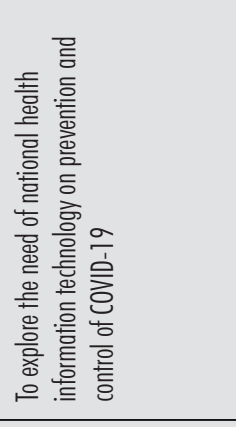 & 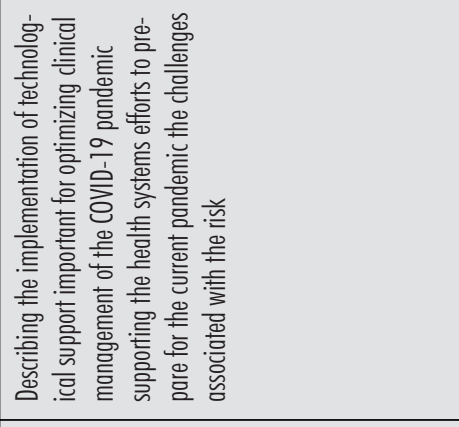 & 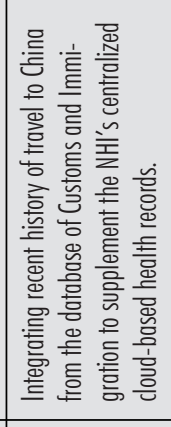 & 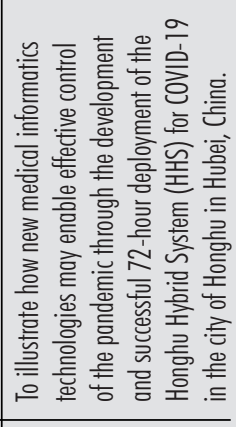 \\
\hline & 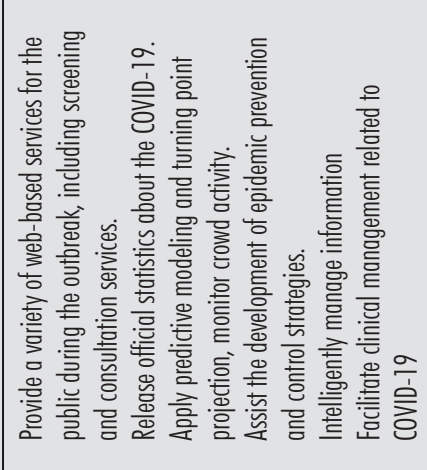 & & 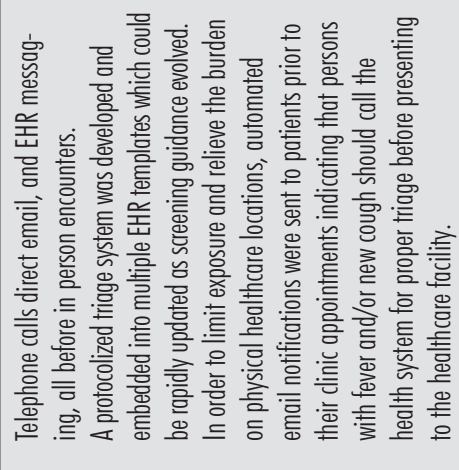 & 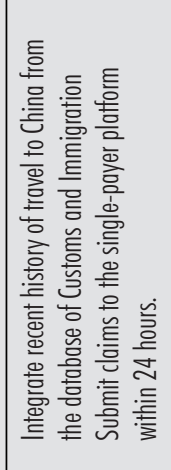 & 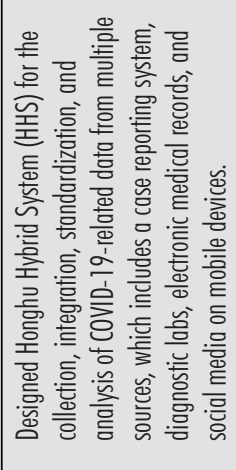 \\
\hline 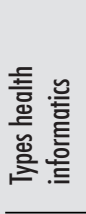 & 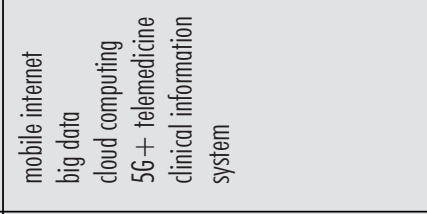 & 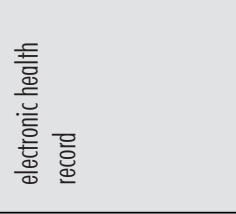 & 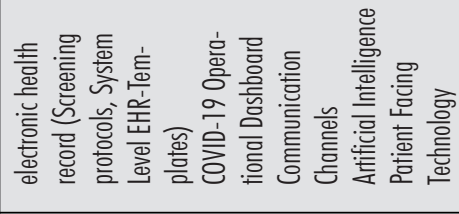 & 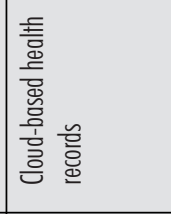 & 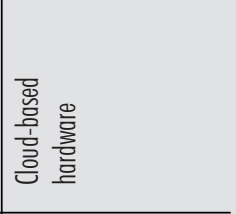 \\
\hline 言 & 吕 & $\underline{\underline{S}}$ & $\underline{\underline{S}}$ & 喜 & 总 \\
\hline 毫 & $1 \cong$ & 套 & 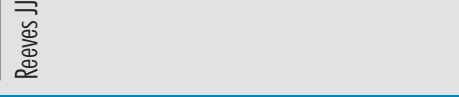 & $\mid \breve{\Xi}$ & $\mid \bar{z}$ \\
\hline
\end{tabular}




\begin{tabular}{|c|c|c|c|c|}
\hline 鮚 & 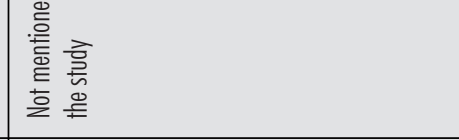 & 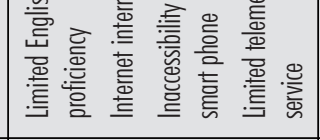 & 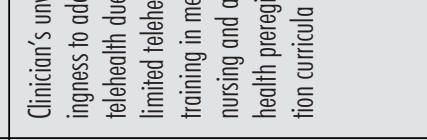 & \\
\hline $\begin{array}{l}\text { 鄫 } \\
\text { 毫 } \\
\text { 言 } \\
\end{array}$ & 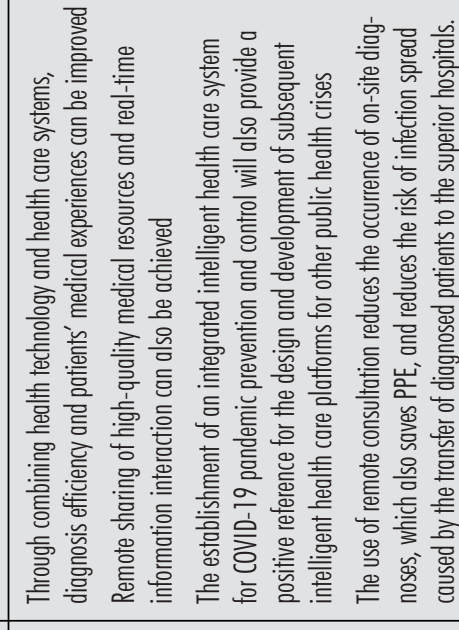 & 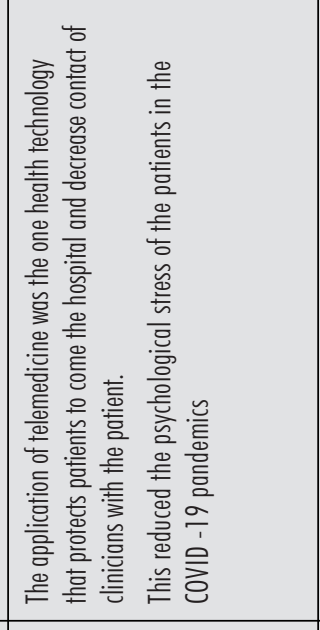 & 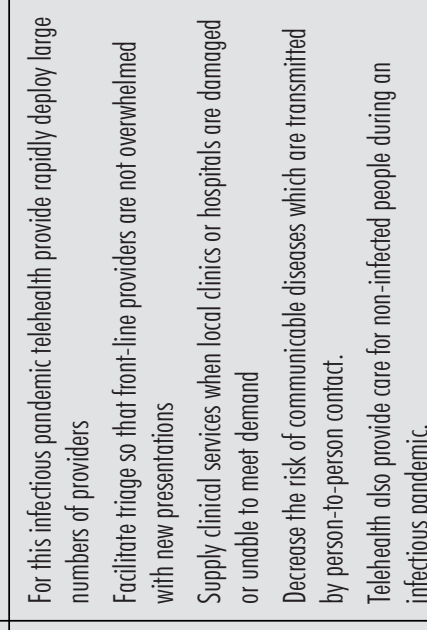 & 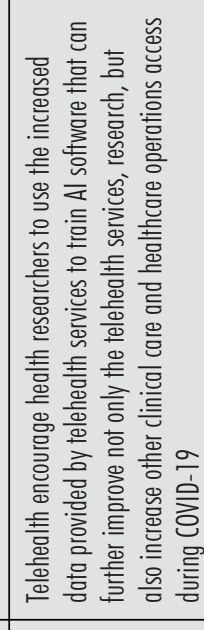 \\
\hline & 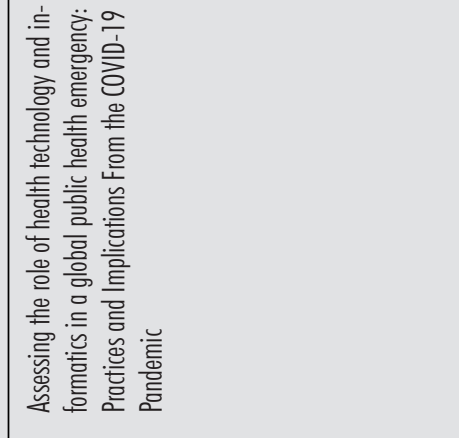 & 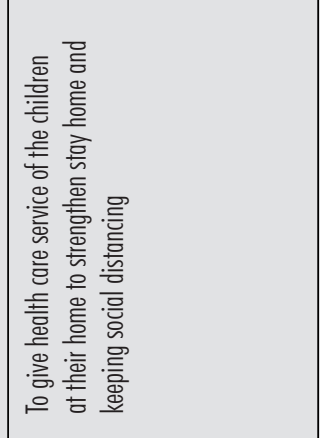 & 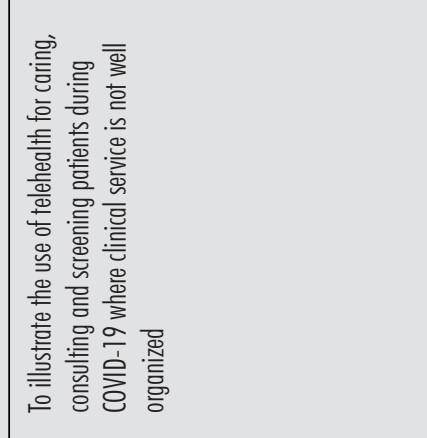 & 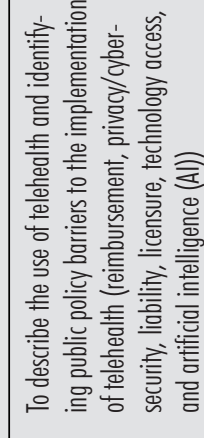 \\
\hline 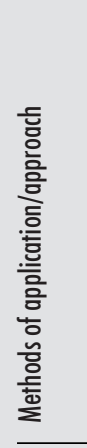 & 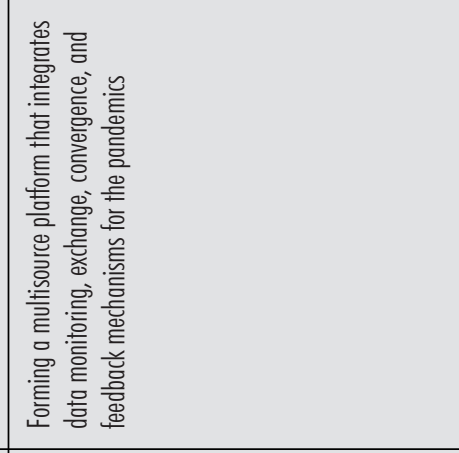 & 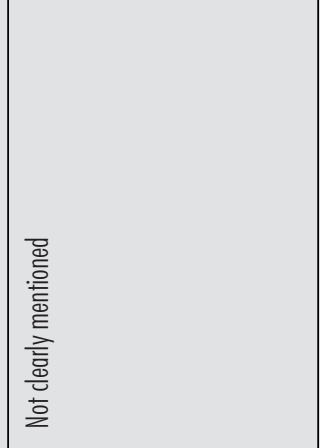 & 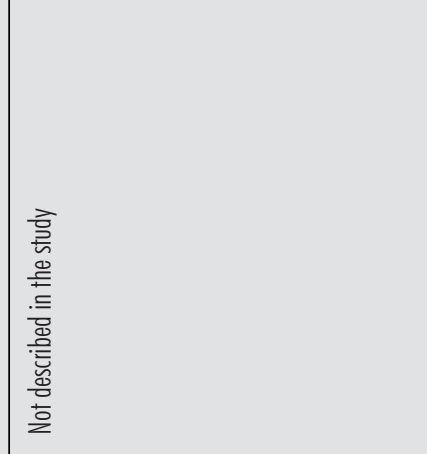 & 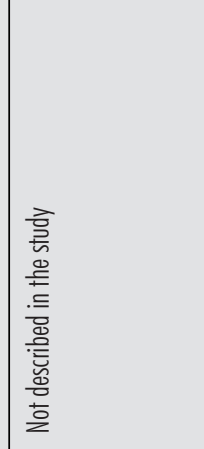 \\
\hline 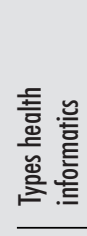 & 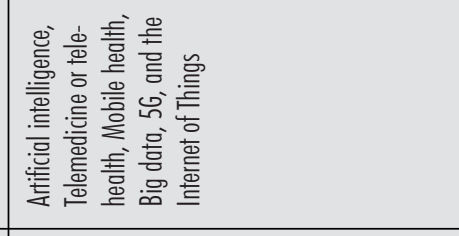 & 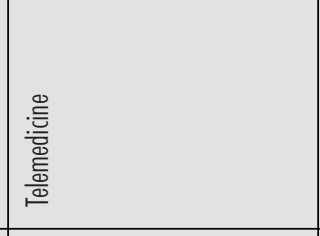 & 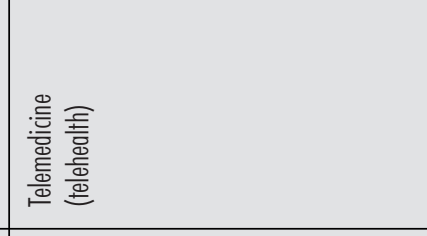 & 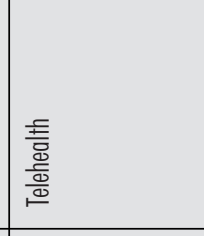 \\
\hline 言 & 孚 & 恐 & 爰 & 永 \\
\hline 毫 & 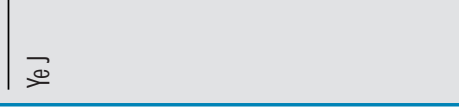 & 善主 & 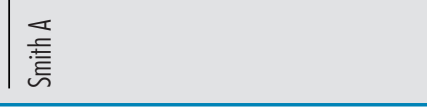 & \begin{tabular}{|l} 
言 \\
高 \\
\end{tabular} \\
\hline
\end{tabular}




\begin{tabular}{|c|c|c|c|c|c|c|}
\hline 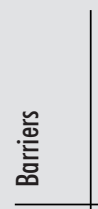 & 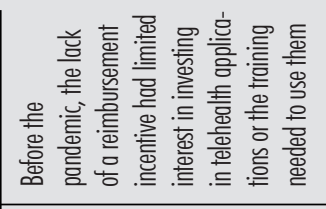 & 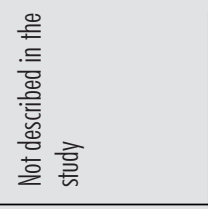 & 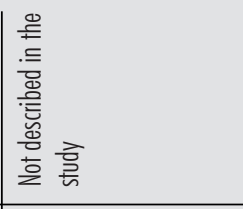 & & 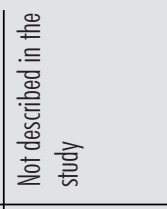 & 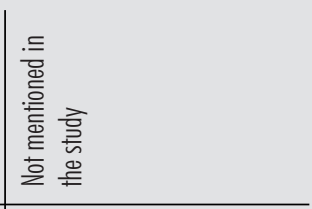 \\
\hline 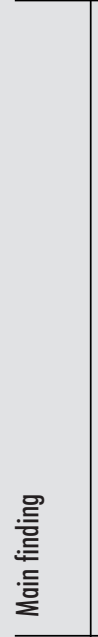 & 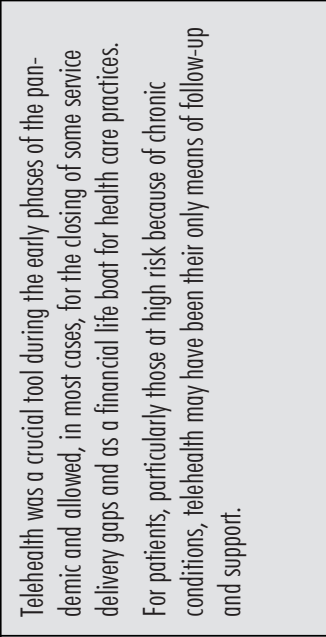 & 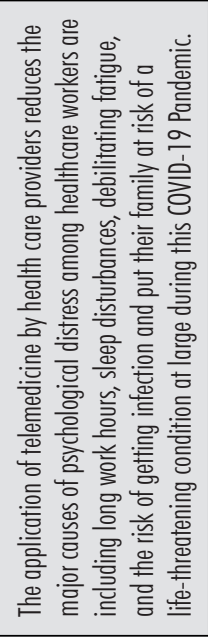 & 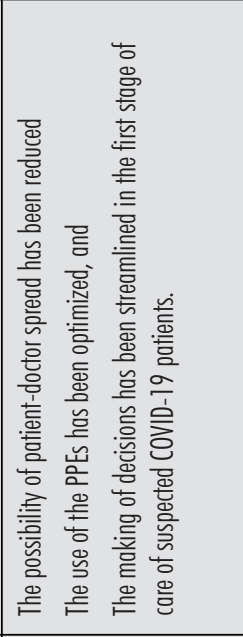 & 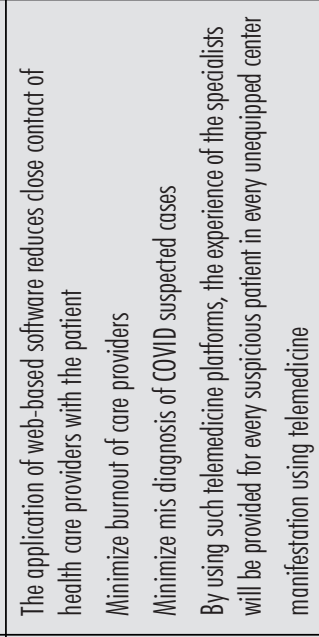 & 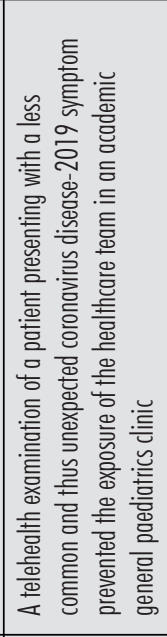 & 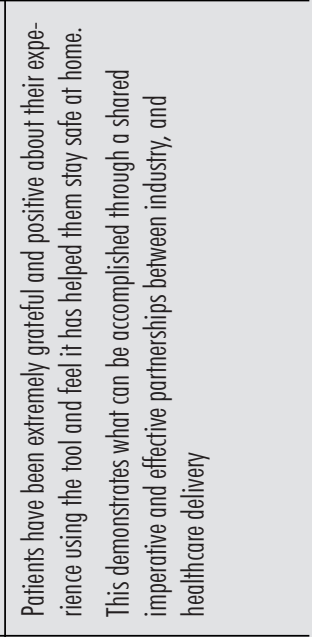 \\
\hline $\begin{array}{l}\text { o. } \\
\text { 产 } \\
\text { 言 }\end{array}$ & 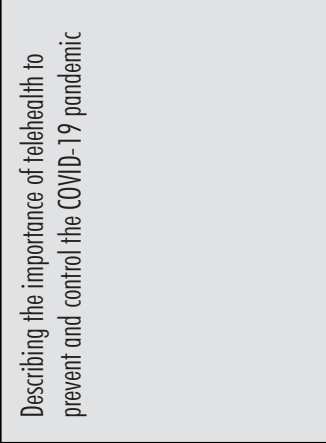 & 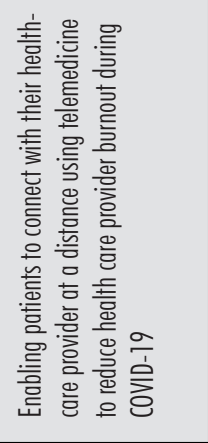 & 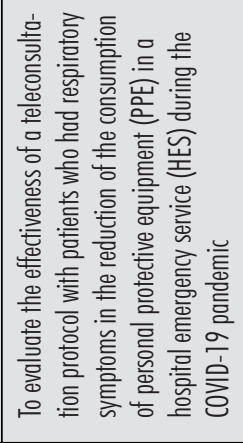 & 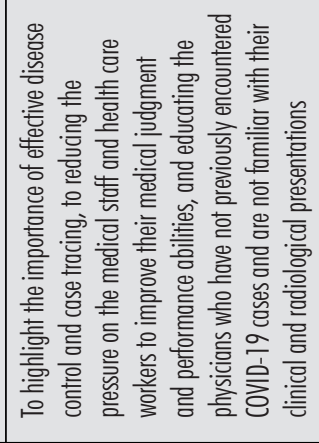 & 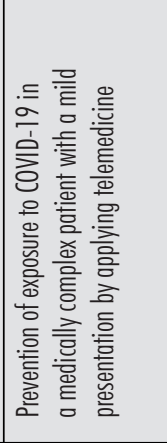 & 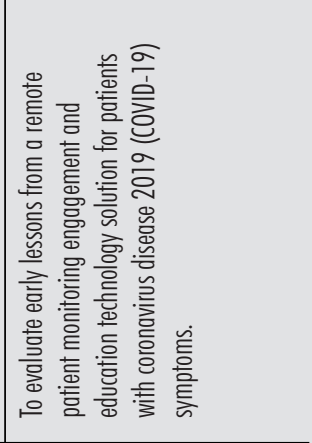 \\
\hline 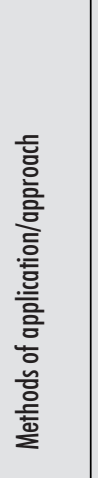 & 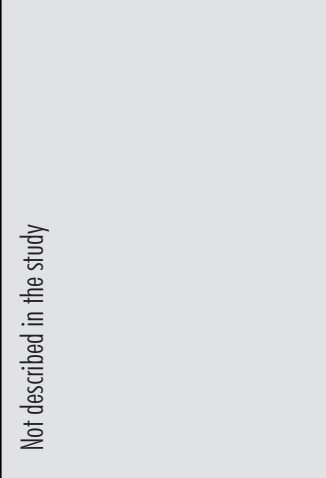 & 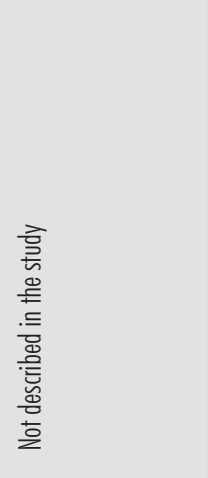 & 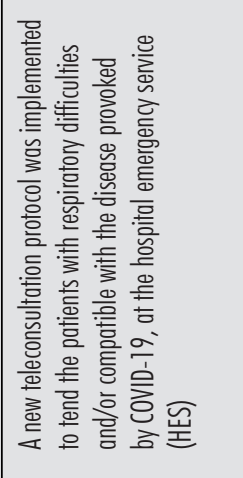 & 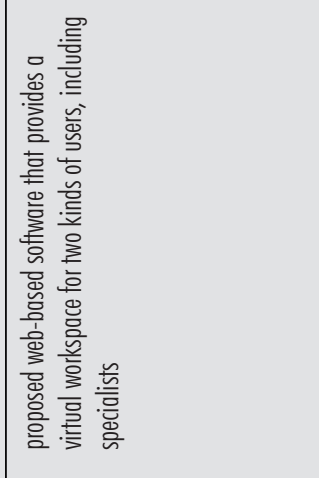 & & 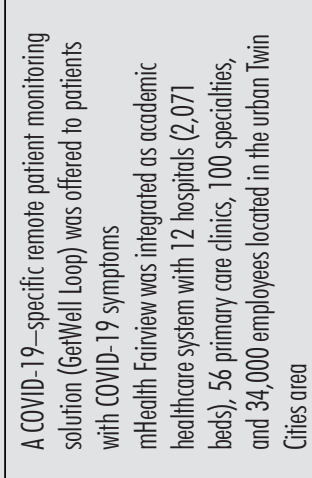 \\
\hline 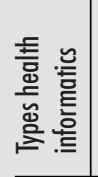 & & 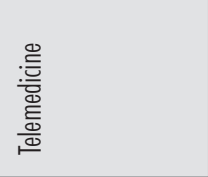 & 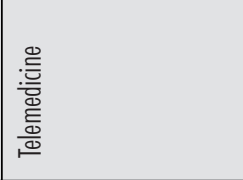 & 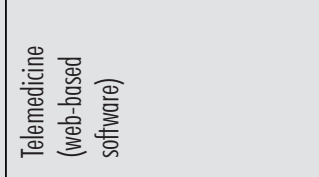 & & 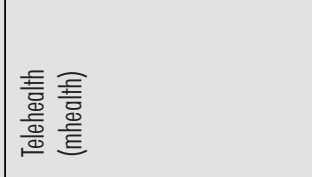 \\
\hline 츨 & 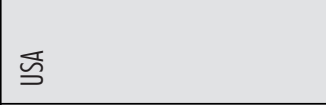 & 흐 & 言 & 흐 & 壱 & 至 \\
\hline 毫 & 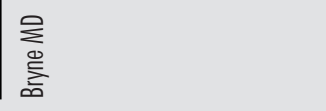 & $\begin{array}{l}\overline{\bar{E}} \\
\text { 品 }\end{array}$ & 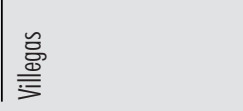 & 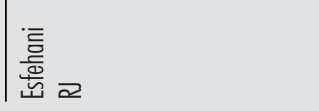 & $\mid \begin{array}{l}\text { 商 } \\
\text { 总 }\end{array}$ & |. \\
\hline
\end{tabular}




\begin{tabular}{|c|c|c|c|c|}
\hline 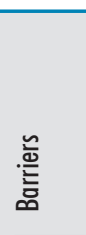 & 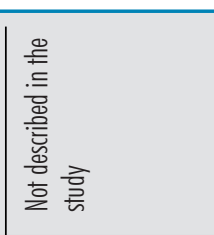 & 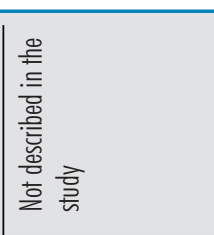 & 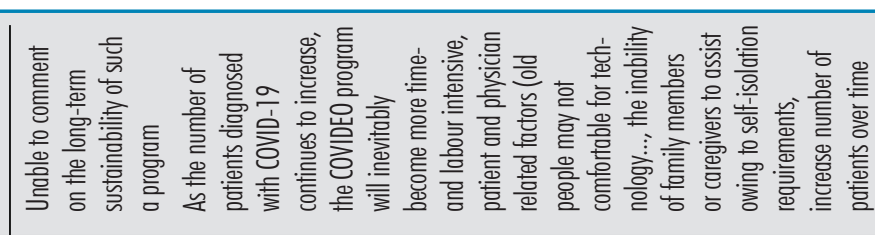 & 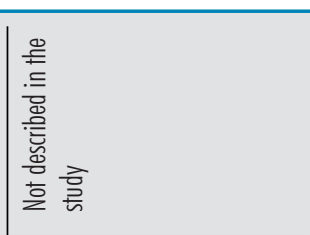 \\
\hline $\begin{array}{l}\text { 墨 } \\
\text { 言 }\end{array}$ & 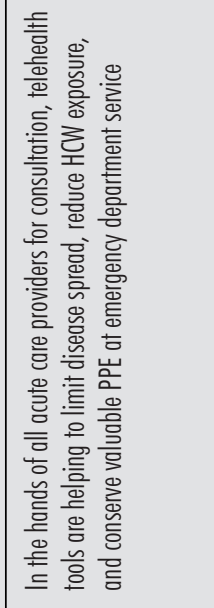 & 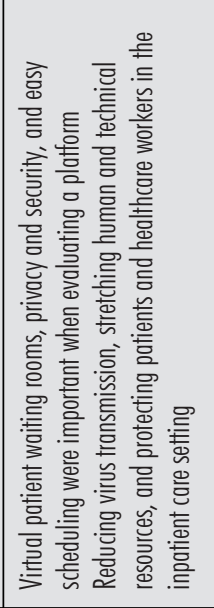 & 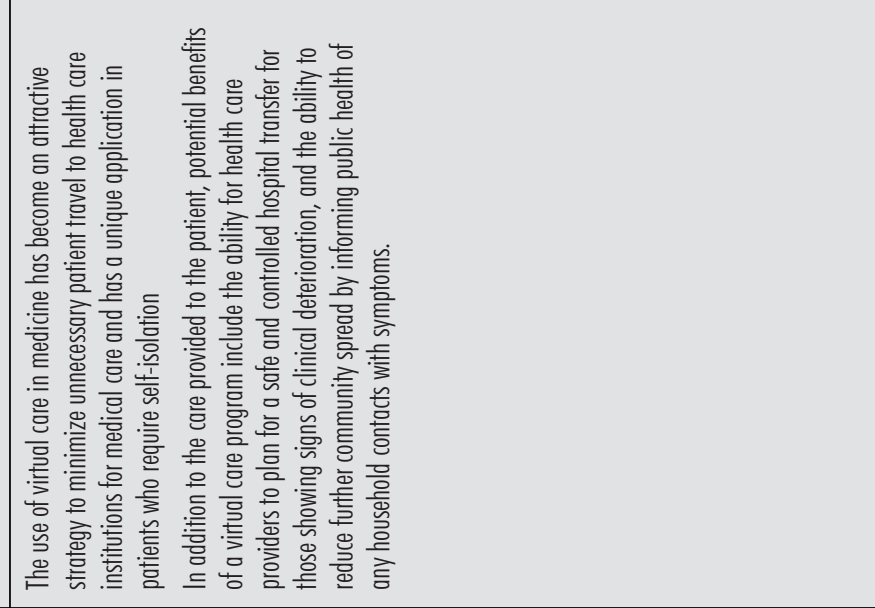 & 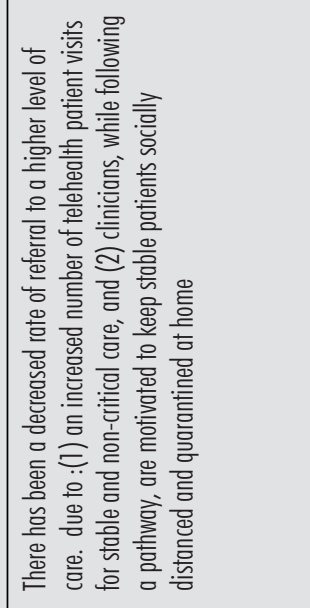 \\
\hline & 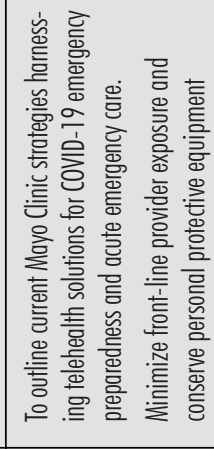 & 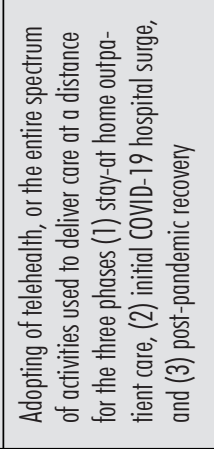 & 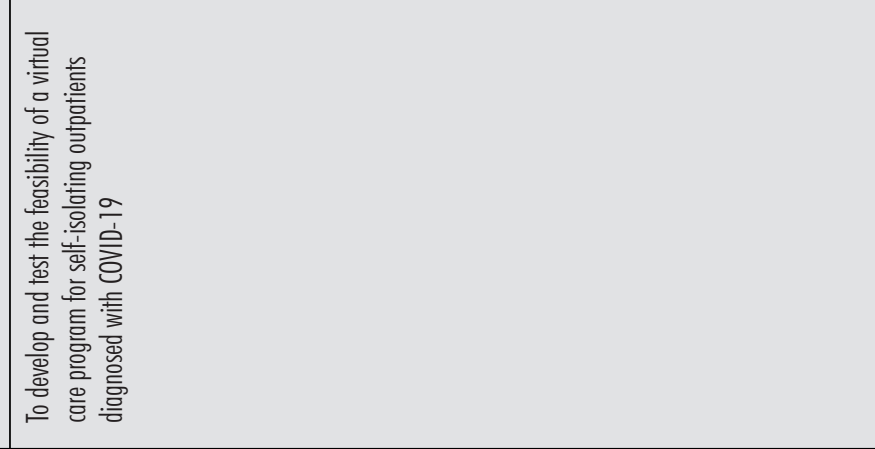 & 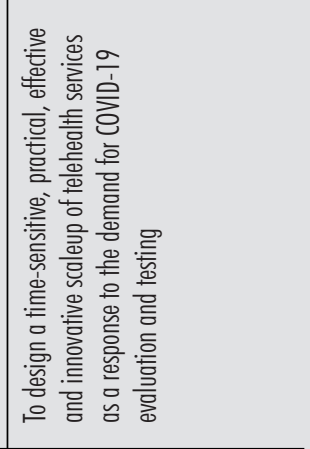 \\
\hline & 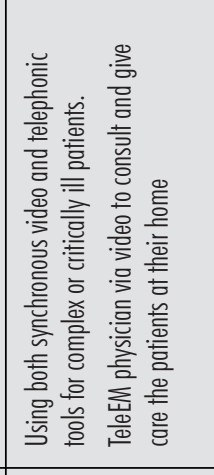 & 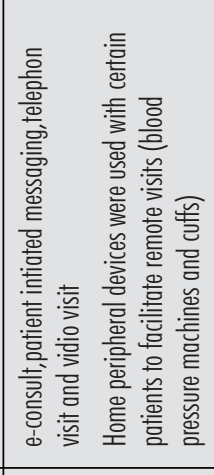 & 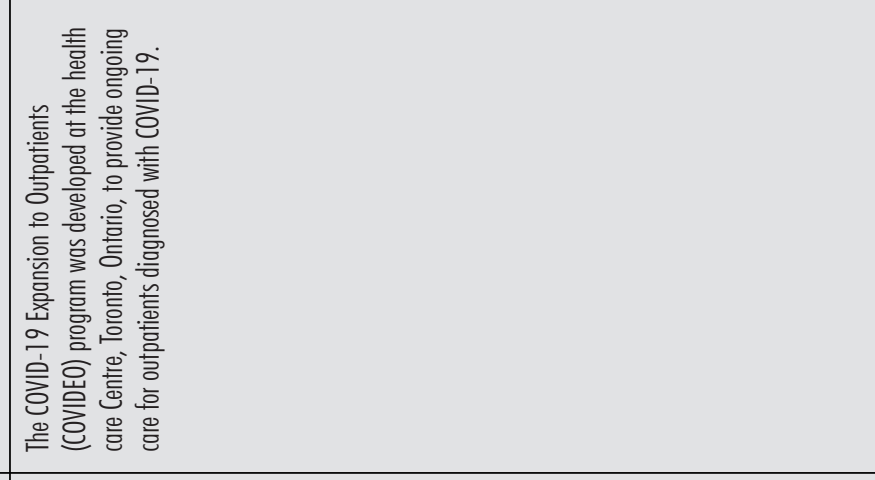 & 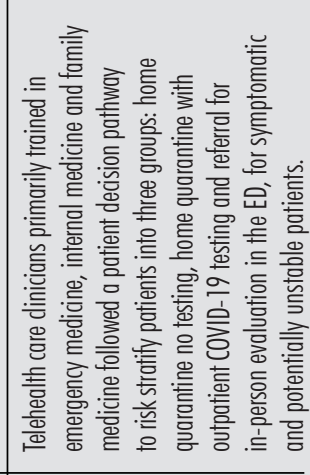 \\
\hline 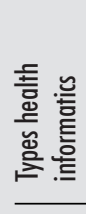 & 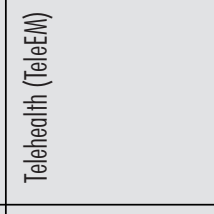 & 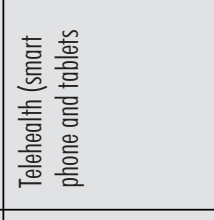 & 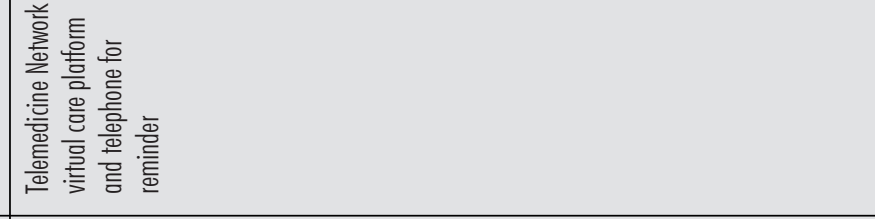 & 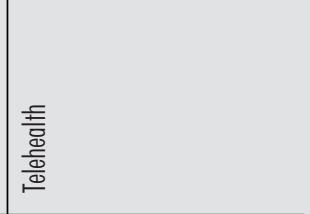 \\
\hline $\begin{array}{l}\text { 言 } \\
\text { 吾 }\end{array}$ & 丣 & $\underline{\underline{\Xi}}$ & 曾 & 祭 \\
\hline 言 & 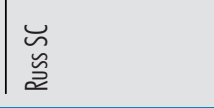 & 咅 & | & $\begin{array}{l}\text { 疍 } \\
\text { 咅 }\end{array}$ \\
\hline
\end{tabular}




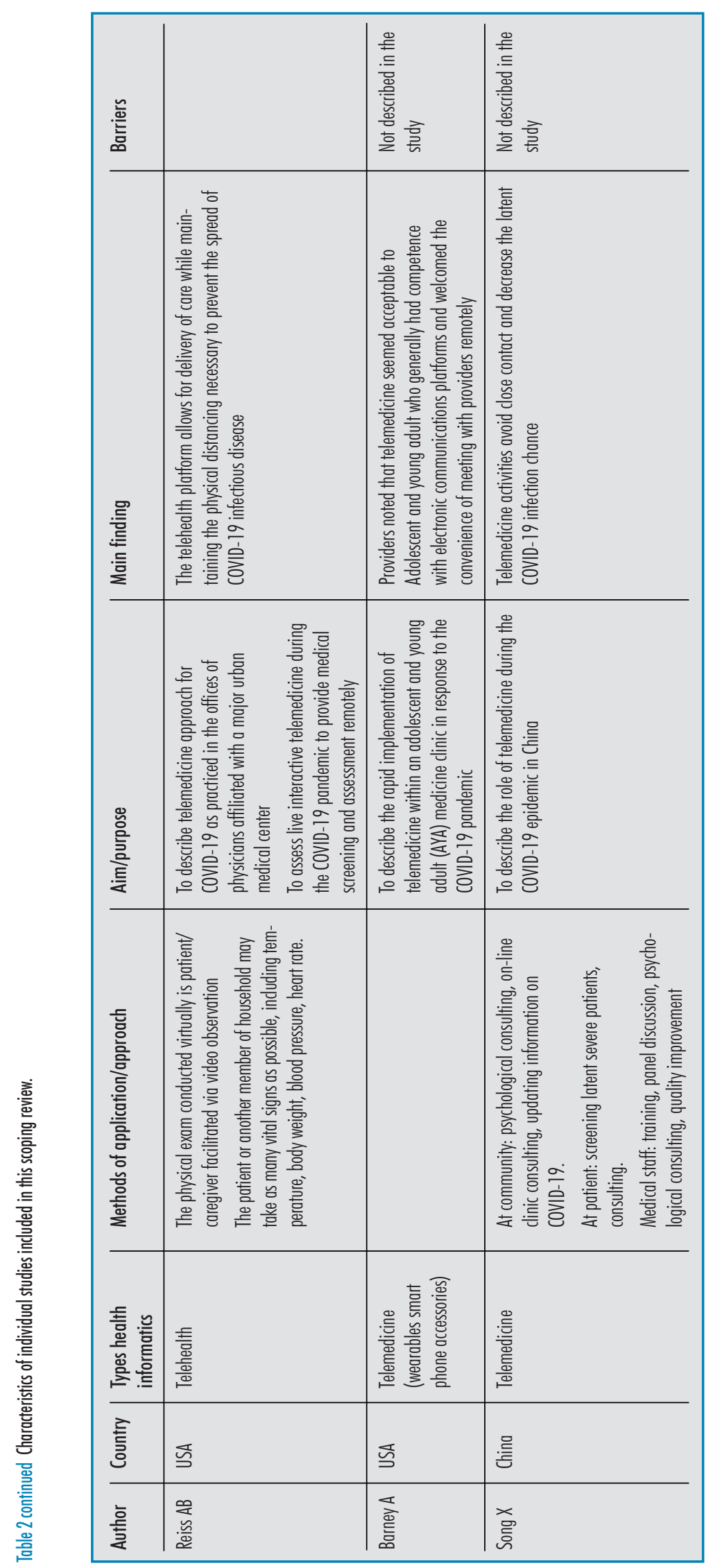




\subsection{What Is the Available Evidence for the Role of Digital Health Technologies for Preventing and Controlling Coronavirus Pandemic?}

\subsubsection{Purpose of Digital Health Technologies} Applied for COVID-19 Pandemic

Different digital health technologies were applied by health care providers for different purposes to prevent and control the COVID-19 pandemic at the community and health care facility levels. These technologies were categorized as telemedicine, Electronic Health Record, Artificial Intelligence, and Internet of Things. In this scoping review, the most frequently used technologies were telemedicine, including telehealth and mHealth [6, 15-24, 27-31], and electronic health records $[13,14,26,32]$. Less frequently used technologies include artificial intelligence $[6,13], 5 \mathrm{G}+[6,25]$, and the Internet of Things [6] (Table 2).

\subsubsection{Telemedicine and mHealth}

Physicians, infectious disease epidemiologists, medical students, and others used telemedicine-based technologies to manage care and to prevent and control the COVID-19 pandemic in the following ways:

- To give health care to the children in their home and to support stay-home orders and to maintain social distancing [15];

- To care for, consult with, and to screen patients during the COVID-19 pandemic where clinical service is not well organized [16] ;

- To identify barriers to the implementation of telehealth during the control of the COVID-19 pandemic [17];

- To enable patients to connect with their healthcare provider at a distance using telemedicine to reduce health care provider burnout during COVID-19 [29] ;

- To evaluate the effectiveness of a teleconsultation protocol with patients who had respiratory symptoms in the face of reduction of the availability of personal protective equipment in a hospital emergency service during the COVID-19 pandemic [30];

- To highlight the importance of effective disease control and contact tracing, to reducing the pressure on the medical staff and health care workers, to improve their medical judgment and performance abilities, and to educate physicians and other health professionals who have not previously encountered COVID-19 cases and who are not familiar with the clinical and radiological presentations of the disease [28];

- To outline current health care strategies for harnessing telehealth solutions for COVID-19 emergency preparedness and acute emergency care and minimize frontline provider exposure and to conserve personal protective equipment [21];

- To adopt telehealth for the entire spectrum of activities used to deliver care at a distance for the three phases of stayat-home: (1) outpatient care, (2) initial COVID-19 hospital surge, and (3) postCOVID recovery [22];

- To develop and test the feasibility of a virtual care program and automated e-mail notifications for self-isolating outpatients diagnosed with COVID-19 [31];

- To assess live, interactive telemedicine during the COVID-19 pandemic to provide remote medical screening and assessment [24].

\subsubsection{Electronic Health Records (EHRs)}

Countries used EHRs for different purposes. These included:

- Using cloud-based or locally maintained EHRs to document and integrate the recent history of travel from customs and immigration databases to supplement, in turn, centralized cloud-based health records maintained by national health institutes [13, 32];

- Using EHRs to explore the need for national digital health technology on prevention and control of COVID-19 and to illustrate how new digital information technologies can enable effective control of the pandemic, such as realized in the development and successful 72-hour deployment of the Honghu Hybrid System for COVID-19 in the city of Honghu in Hubei [14, 26];

- Creating a protocolized triage system was developed and embedded into multiple EHR templates which could be rapidly updated as screening guidance evolved [13]. Another example was the Honghu Hybrid System for the collection, integration, standardization, and analysis of COVID-19-related data from multiple sources, which includes a case reporting system, diagnostic labs, electronic medical records, and social media on mobile devices [26].

\subsubsection{Artificial Intelligence (AI) and Internet of Things (loT)}

To manage, prevent, and control the COVID-19 pandemic; health care providers used $\mathrm{AI}$ and IoT for the following purposes:

- To understand the actions taken by the health informatics community in China during the COVID-19 outbreak and developing a digital health technology framework for epidemic response based on digital health technology-related measures and methods by using IoT [25];

- To support health system efforts to prepare for the current pandemic challenges associated with the risk by using AI [13];

- To assess the role of digital health technology in a global Public Health Emergency by using both AI and IoT [6];

- Managing information and facilitating clinical management related to COVID-19 including screening and consultation services, predicting modelling and turning point projection (6)

\subsection{What Was the Additional Importance of Using Digital Health Technologies for the Prevention and Control of COVID-19?}

\subsubsection{Exchanging Health Information and Minimizing Pandemic Exposure}

The applications of DHTs had contributed to exchanging information and preventing exposure of health care providers and patients to COVID-19. Telemedicine activities avoid close contact and decrease the latent COVID-19 infection chance, limit disease spread, reduce health care workers exposure, minimize patient-doctor spread, and help to streamline decisions made in the first stage of care of suspected COVID-19 patients [21, 
27]. Patient-facing telemedicine platforms included a COVID-19 information page, which updated the latest information in real-time, including providing community patients with symptom information through online clinic consultation services, instructions for quarantine processes at home, personal protection applications, and when to seek medical attention $[19,21,27,30]$.

\subsubsection{Early Test, Isolation, and Treatment During the Outbreak}

Through combining health technology and health care systems, remote sharing of high-quality medical resources and real-time information interaction can be achieved [6]. In addition, diagnostic efficiency and disease course can be improved. Using electronic health record components such as screening protocols, system-level EHR-templates, COVID-19 operational dashboards, and communication channels, rapid screening processes, and laboratory testing, clinical decision support, and patient-facing technology related to COVID-19 were achieved [6, 13]. Digital health technologies such as telemedicine network virtual care platforms and cloud-based hardware helped strengthen the checkpoints on the full chain of COVID-19 control, including "early test, early report, early isolation, and early treatment" during the outbreak [25, 28, 31].

\subsubsection{Remote Consultation and Reducing Psychological Stress}

The application of telemedicine by health care providers reduces the major causes of psychological distress among healthcare workers by reducing long work hours, sleep disturbances, debilitating fatigue, and fear of getting an infection that could also put their family at risk of infection. Furthermore, it protects patients who otherwise might come to the hospital and thus decreases contact with health care providers [15, 29].

\subsubsection{Reduce Crowding of Health Service and Care Provider Burnout}

Telehealth was a crucial tool during the COVID-19 pandemic and allowed in most cases, for the closing of some service de- livery gaps and as a financial lifeboat for health care practices [17]. It also increased other clinical care and healthcare operations access during COVID-19 [17, 18]. Developing telemedicine platforms by using web-based software reduced close contact of health care providers with the patient, minimized exhaustion and burnout of care providers, and minimized misdiagnosis of COVID suspected cases [23, 28].

\subsubsection{Digital Health Technologies to Assist Epidemiologists and Infectious Disease Professionals}

In addition to the specific types of digital health information technology modalities described above, our review found that the application of digital health technologies (DHTs) was important to controlling and preventing the COVID-19 pandemic. A study in China revealed that DHTs were effective for the prediction of epidemic trends, tracking of close contacts, and remote diagnosis of COVID-19 pandemics. This will help public health decision-makers to develop COVID-19 pandemic prevention and control strategies $[33,34]$. Additionally, these technologies will facilitate effective COVID-19 vaccine delivery by tracking vaccine individuals, managing the data, and maintaining the cold chain [34].

\subsection{What Were the Barriers That Influence the Practice of Digital Health Technologies During COVID-19 Prevention and Control of the Pandemic?}

The COVID-19 pandemic has had an impact on public health infrastructure necessitating the development and use of digital health technologies. The need to frequently adjust this technology to meet rapidly evolving requirements, communication and adoption to coordinate the needs of multiple stakeholders for controlling and management of the pandemic pushed the need to use digital technologies even further. During the period covered by these reviews, some barriers reduced the effec- tiveness of the prevention of COVID-19. These barriers are mainly centered in three categories: patients, health care providers, and financial issues [16, 18, 25].

Some of the commonest barriers were: clinician's unwillingness to adopt telehealth due to limited telehealth training in medical, nursing and allied health pre-registration curricula [16], the economic incentive for doctors (doctors and their staffs time), and for system integrators and device manufacturers to develop the technology [17]. The old people were not also comfortable with technologies, the inability of family members or caregivers to assist owing to self-isolation requirements, increase the number of COVID-19 case over time, the lack of a reimbursement incentive, and economic incentives for health care providers. Additionally, limited English proficiency, internet service interruption, and inaccessibility of smartphones were the barriers of digital technologies service to be effective for controlling and preventing the pandemics $[15,18,31]$.

Various papers revealed that there are different barriers to apply DHTs properly; such as lack of technical supporter, interference with a patient-provider relationship, increased patient anxiety, cost of digital health equipment, and lack of integration of technology with clinical workflow $[35,36]$.

The current review showed that limited English proficiency of the patients, internet interruption, inaccessibility of smartphone, limited telehealth training for health care providers were some barriers reduced the effectiveness of the digital health technologies. This is also supported by a WHO review in which disparities in digital literacy and access to equipment, broadband, and the internet are important for increasing the effectiveness of telemedicine on COVID- 19 pandemic prevention [37]. This indicates that there is a need for immediate action by responsible stakeholders to facilitate the application of electronic technologies to reduce the burden of the pandemics and to recover the normal life of the world people especially countries which do not have good health care system [38-40]. 


\section{Discussion}

Our aim in this review was three-fold: 1) to map the role of digital health technologies on prevention and control of the COVID-19 pandemic; 2) to understand how the digital technologies have been applied to prevent the pandemic; and 3) to investigate the main barriers to the use of these technologies for tracking the pandemic.

About two-thirds of published studies on digital technologies for preventing and controlling the COVID-19 pandemic were from North America followed by Asia. Furthermore, we found that there were no published studies from Africa and limited studies from Europe and Australia. Our review found that the most commonly used DHTs to track COVID-19 pandemics were: telehealth, mHealth, EHRs, AI, and IoT. This finding is supported by prior analyses in which these digital health technologies were used to control and manage the Ebola and Zika [41, 42] pandemics using similar approaches.

We found that the internet provides a variety of web-based services for the public during the outbreak, including video consultation, screening, and diagnosis. These services support the health care provider to identify a suspected case of COVID-19 and to provide medical and psychological advice to patients with the disease. Our review found that health care providers applied DHTs using different approaches for preventing and controlling coronavirus 2019. For instance, online clinic consultation, updating information on COVID-19 for the community, video and telephone consultation, and a multi-source platform that integrated data monitoring, exchange, and feedback mechanisms for pandemics-related issues.

This review suggests that digital health technologies like telehealth, EHRs, AI, IoT, and telemedicine platforms contributed to reducing COVID-19 exposure and transmission from person to person by exchanging health information, early testing and diagnosis, early isolation, early treatment during the outbreak, and remote consultation. This potentially helps to reduce burnout of health care providers, psychological stress, and overcrowding of health facilities. Also, it facilitates real-time information interaction and provides support for data-driven decision-making in the time of this pandemic and increasing susceptibility to COVID-19 especially for the elderly and those with comorbid conditions $[9,43]$.

As we have seen, elderly people who might not be comfortable with technologies, the inability of family members or caregivers to assist owing to self-isolation requirements, increase the number of COVID-19 case over time, the lack of a reimbursement incentive, and economic incentives for health care providers are the most common barriers that decreased the effectiveness of DHTs application for prevention and control of COVID-19.

To expand the DHTs for the next pandemic, it is recommended that providing a variety of web-based services for the public during the outbreak, including screening and consultation service; forming a multisource platform that integrates data monitoring, exchange, convergence, and feedback mechanisms for the pandemics-related parties, and offering COVID-19 specific remote patient monitoring solutions.

Though this review uses a comprehensive search strategy from various databases, scoping reviews are broad and provide an overview of existing literature regardless of quality, providing a broad and contextual overview in comparison to systematic reviews. A formal assessment of methodological quality is not undertaken when conducting a scoping review and synthesis of the incorporating studies, nor is a demonstration of cause-and-effect relationships. Excluding grey literature and languages other than English are additional limitations of the review.

\section{Conclusion}

The current scoping review showed that using DHTs are effective in the prevention and control of the COVID-19 pandemic. These digital health technologies are principally used for the early test, report, isolation, treatment, exchanging information, remote consulting, reducing the overcrowding of health care facilities, minimizing burnout of health care providers, and model prediction
COVID-19. The lessons learned during the COVID-19 pandemic should be applied to future digital health technologies that seek to support health system response to a pandemic.

\section{Acknowledgments}

The authors would like to acknowledge Dr. Brian Dixon and Dr. John Holmes for their support throughout the production of this review article.

\section{References}

1. Fagherazzi G, Goetzinger C, Rashid MA, Aguayo GA, Huiart L. Digital health strategies to fight COVID-19 worldwide: challenges, recommendations, and a call for papers. J Med Internet Res 2020;22(6):e19284.

2. Wilson C, Jumbert MG. The new informatics of pandemic response: humanitarian technology, efficiency, and the subtle retreat of national agency. Journal of International Humanitarian Action 2018:3(1):1-13.

3. Mantas J, Hasman A, Househ M. The Importance of Health Informatics in Public Health during a Pandemic. IOS Press; 2020.

4. Ahmadi S, Bempong N-E, De Santis O, Sheath D, Flahault A. The role of digital technologies in tackling the Zika outbreak: a scoping review. J Public Health Emerg 2018;2(6).

5. Wood CS, Thomas MR, Budd J, Mashamba-Thompson TP, Herbst K, Pillay D, et al. Taking connected mobile-health diagnostics of infectious diseases to the field. Nature 2019;566(7745):467-74.

6. Ye J. The role of health technology and informatics in a global public health emergency: practices and implications from the COVID-19 pandemic. JMIR Med Inform 2020;8(7):e19866.

7. Wang S, Ding S, Xiong L. A New System for Surveillance and Digital Contact Tracing for COVID-19: Spatiotemporal Reporting Over Network and GPS. JMIR MHealth UHealth 2020;8(6):e19457.

8. Kahn JP. Digital contact tracing for pandemic response: Ethics and governance guidance. Johns Hopkins University Press; 2020.

9. Cassione EB, Zanframundo G, Biglia A, Codullo V, Montecucco C, Cavagna L. Response to: 'COVID-19 pandemic: an opportunity to assess the utility of telemedicine in patients with rheumatic diseases' by Lopez-Medina et al. Ann Rheum Dis 2020.

10. Peden CJ, Mohan S, Pagán V. Telemedicine and COVID-19: an Observational Study of Rapid Scale Up in a US Academic Medical System. J Gen Intern Med 2020 Sep;35(9):2823-5.

11. Peters M, Godfrey C, Khalil H, McInerney P, Soares C, Parker D. Guidance for the Conduct of JBI Scoping Reviews. 2017.

12. Arksey H, O'Malley L. Scoping studies: towards a methodological framework. Int J Soc Res Meth- 
odol 2005;8(1):19-32.

13. Reeves JJ, Hollandsworth HM, Torriani FJ, Taplitz $\mathrm{R}$, Abeles S, Tai-Seale M, et al. Rapid response to COVID-19: health informatics support for outbreak management in an academic health system. J Am Med Inform Assoc 2020;27(6):853-9.

14. Sittig DF, Singh H. COVID-19 and the Need for a National Health Information Technology Infrastructure. JAMA 2020 Jun 16;323(23):2373-4.

15. Katzow MW, Steinway C, Jan S. Telemedicine and Health Disparities During COVID-19. Pediatrics 2020; Aug;146(2):e20201586.

16. Smith AC, Thomas E, Snoswell CL, Haydon H, Mehrotra A, Clemensen J, et al. Telehealth for global emergencies: Implications for coronavirus disease 2019 (COVID-19). J Telemed Telecare 2020 Jun;26(5):309-3.

17. Hoffman DA. Increasing access to care: telehealth during COVID-19. J Law Biosci 2020;7(1):Isaa043.

18. Byrne MD. Telehealth and the COVID-19 Pandemic. J Perianesth Nurs 2020;35(5):548-51.

19. Chavis A, Bakken H, Ellenby M, Hasan R. COVID-19 and Telehealth: Prevention of Exposure in a Medically Complex Patient With a Mild Presentation. J Adolesc Health 2020;67(3):456-8.

20. Annis T, Pleasants S, Hultman G, Lindemann E, Thompson JA, Billecke S, et al. Rapid Implementation of a COVID-19 Remote Patient Monitoring Program. J Am Med Inform Assoc 2020 Aug 1;27(8):1326-30.

21. Russi CS, Heaton HA, Demaerschalk BM, editors. Emergency Medicine Telehealth for COVID-19: Minimize Front-Line Provider Exposure and Conserve Personal Protective Equipment. Mayo Clinic Proceedings. Elsevier; 2020.

22. Wosik J, Fudim M, Cameron B, Gellad ZF, Cho A, Phinney D, et al. Telehealth Transformation: COVID-19 and the rise of Virtual Care. JAm Med Inform Assoc 2020;27(6):957-62.

23. Joshi AU, Lewiss RE. Telehealth in the time of COVID-19. Emerg Med J 2020;37(10):637-8.

24. Reiss AB, De Leon J, Dapkins IP, Shahin G, Peltier MR, Goldberg ER. A Telemedicine Approach to Covid-19 Assessment and Triage. Medicina (Kaunas) 2020;56(9):461.

25. Ye Q, Zhou J, Wu H. Using Information Technology to Manage the COVID-19 Pandemic: Development of a Technical Framework Based on
Practical Experience in China. JMIR Med Inform 2020;8(6):e19515.

26. Gong M, Liu L, Sun X, Yang Y, Wang S, Zhu H. Cloud-Based System for Effective Surveillance and Control of COVID-19: Useful Experiences From Hubei, China. J Med Internet Res 2020;22(4):e18948.

27. Song X, Liu X, Wang C. The role of telemedicine during the COVID-19 epidemic in China-experience from Shandong province. Crit Care 2020 Apr 28;24(1):178.

28. Jafarzadeh-Esfehani R, Fard MM, Hatam-Ghale FH, Kalat AR, Fathi A, Shariati M, et al. Telemedicine and Computer-Based Technologies during Coronavirus Disease 2019 Infection; A Chance to Educate and Diagnose. Arch Iran Med 2020;23(8):561-3.

29. Moazzami B, Razavi-Khorasani N, Moghadam AD, Farokhi E, Rezaei N. COVID-19 and telemedicine: Immediate action required for maintaining healthcare providers well-being. J Clin Virol 2020:104345.

30. Lopez-Villegas A, Maroto-Martin S, Baena-Lopez MA, Garzon-Miralles A, Bautista-Mesa RJ, Peiro $\mathrm{S}$, et al, editors. Telemedicine in Times of the Pandemic Produced by COVID-19: Implementation of a Teleconsultation Protocol in a Hospital Emergency Department. Healthcare (Basel) 2020 Sep 23;8(4):357.

31. Lam PW, Sehgal P, Andany N, Mubareka S, Simor $\mathrm{AE}$, Ozaldin $\mathrm{O}$, et al. A virtual care program for outpatients diagnosed with COVID-19: a feasibility study. CMAJ Open 2020;8(2):E407-E13.

32. Lin C, Braund WE, Auerbach J, Chou J-H, Teng $\mathrm{J}-\mathrm{H}, \mathrm{Tu}$ P, et al. Policy Decisions and Use of Information Technology to Fight COVID-19, Taiwan. Emerg Infect Dis 2020;26(7):1506-12.

33. Kuniya T. Prediction of the epidemic peak of coronavirus disease in Japan, 2020. J Clin Med 2020;9(3):789.

34. Chretien J-P, Swedlow D, Eckstrand I, George D, Johansson M, Huffman R, et al. Advancing epidemic prediction and forecasting: a new US government initiative. Online J Public Health Inform 2015;7(1).

35. Inkster B, O'Brien R, Selby E, Joshi S, Subramanian V, Kadaba M, et al. Digital Health Management During and Beyond the COVID-19 Pandemic:
Opportunities, Barriers, and Recommendations. JMIR Ment Health 2020 Jul 6;7(7):e19246.

36. Palacholla RS, Fischer N, Coleman A, Agboola S, Kirley K, Felsted J, et al. Provider-and patient-related barriers to and facilitators of digital health technology adoption for hypertension management: scoping review. JMIR Cardio 2019;3(1):e11951.

37. Greenhalgh T, Wherton J, Shaw S, Morrison C. Video consultations for covid-19. BMJ $2020 \mathrm{Mar}$ 12;368:m998.

38. Alwashmi MF. The use of digital health in the detection and management of COVID-19. Int J Environ Res Public Health 2020;17(8):2906.

39. Hageman JR. The emergence of pediatric telehealth as a result of the COVID-19 pandemic. Pediatric Ann 2020;49(7):e283-e284.

40. Castelnuovo G, Pietrabissa G, Manzoni GM, Sicurello F, Zoppis I, Molinari E. Fighting the COVID-19 pandemic using the technology-based second-line in Italy and Lombardy: The urgent need of home-based remote monitoring systems to avoid the collapse of the hospital-centred first line. J Global Health 2020 Dec;10(2):010371.

41. Khubchandani J, Jordan TR, Yang YT. Ebola, Zika, Corona... What Is Next for Our World? Int J Environ Res Public Health 2020 May 2;17(9):3171.

42. Sacks JA, Zehe E, Redick C, Bah A, Cowger K, Camara $\mathrm{M}$, et al. Introduction of mobile health tools to support Ebola surveillance and contact tracing in Guinea. Glob Health Sci Pract 2015;3(4):646-59.

43. Carlson JL, Goldstein R. Using the Electronic Health Record to Conduct Adolescent Telehealth Visits in the Time of COVID-19. J Adolesc Health 2020 Aug;67(2):157-8.

Correspondence to:

Binyam Tilahun

Department of Health Informatics

Institute of Public Health

College of Medicine and Health Sciences

University of Gondar

Gondar, Ethiopia

E-mail: Binyam.Tilahun@uog.edu.et 